\title{
Extending Web Browsers Architectures to support HTTP Session Mobility
}

\author{
Michael O. Adeyeye \\ $\mathrm{Mr}$. \\ Department of Electrical Engineering \\ University of Cape Town \\ Private Bag X3, Rondebosch 7701, \\ South Africa. \\ (+27) 737367039 \\ micadeyeye@crg.ee.uct.ac.za
}

\author{
Neco Ventura \\ Mr. \\ Department of Electrical Engineering \\ University of Cape Town \\ Private Bag X3, Rondebosch 7701, \\ South Africa. \\ (+27) 216502084 \\ neco@crg.ee.uct.ac.za
}

\begin{abstract}
In a bid to extend web browsers capabilities, a new extension that transfers HTTP session between two web browsers also knows as User Agents will be developed. The capabilities of these web browsers will be extended by integrating a Session Initiation Protocol (SIP) stack into them. SIP has been chosen because it has clearly defined session mobility types namely Third-party Call Control and Session Hand-off. This paper identifies the modifications that will be made to the present-day web browsers architectures and describes the two services that can be provided namely content sharing and session transfer between any two user agents.
\end{abstract}

\section{Keywords}

Web Browser Architecture, HTTP Session Mobility.

\section{INTRODUCTION}

Though HTTP can be made stateful [1], session continuity between User Agents has been achieved by varying approaches resulting in the need for standardization. Session Initiation Protocol (SIP) is a signaling protocol that has been adopted for the IP Multimedia Subsystem (IMS) by the 3rd Generation Partnership Project (3GPP). It has been extended to provide instant messaging and presence services through SIP Instant Messaging and Presence Leveraging Extensions (SIMPLE) [2]. The intention of this research is to implement HTTP Session Mobility using SIP. Mozilla Firefox web browser will be used to implement the client side of this research because it is FOSS, has rich documentations on the internet and a large community of contributors.

Permission to make digital or hard copies of all or part of this work for personal or classroom use is granted without fee provided that copies are not made or distributed for profit or commercial advantage and that copies bear this notice and the full citation on the first page. To copy otherwise, or republish, to post on servers or to redistribute to lists, requires prior specific permission and/or a fee.

CoNEXT'07, December 10-13, 2007, New York, U.S.A.

Copyright 2007 ACM 978-1-59593-770-4/ 07/ 0012 ...\$5.00

\section{BACKGROUND}

The driving forces behind HTTP Session Mobility using SIP include the following. First, in [4] it was proposed that IMS should integrate HTTP Proxy, Real-time Streaming Transfer Protocol (RSTP) Proxy and Application Policy Function in order to harmonize SIP, HTTP and RTSP service delivery. Second, the major protocols in converged applications are SIP and HTTP. Today, user equipment meant for voice, video and data communication are often pre-built with different user agents for different services.

With the convergence in networks and services, unified user agents or adaptive user agents will be emerging. Typically, a web browser could be used as a telephony client by installing appropriate extension. At present, there is a SIP Client which was developed using Mozilla Gecko [5]. With HTTP Session Mobility, users will be able to change communication modes seamlessly when accessing a multichannel or multimodal application, a process referred to as Terminal Mobility.

\section{RELATED WORK}

HTTP Session Mobility has been carried out using different schemes namely Client, Server and Proxy Architectural Schemes $[6,7,8]$. Each scheme requires modifying the User Agent Client (Web browser) or User Agent Server (Web server) or both. In some cases, a Proxy User Agent could be placed along the communication path of the client and the server, as found in the Proxy Architectural Scheme. In previous works, the identification or authentication mechanism for users was not clearly stated, the session mobility paradigms were not based on standards but personal initiatives and the HTTP 1.1 security as stated in RFC 2616 [9] was broken during one of the implementations [6].

\section{THE PROPOSED HTTP SESSION MOBILITY SERVICE}

In this research, HTTP session mobility using SIP will be achieved by extending the capabilities of a User Agent 
Client and implementing a SIP Application Server which provides HTTP session transfer or content sharing without violating HTTP/1.1 security. This scheme is referred to as a Hybrid-based Architectural Scheme whereby the client and the proxy or application server are modified and improvised respectively. This service can be adapted to the 3G IMS because it also uses SIP for signaling and communication with the application server. The HTTP session transfer and content sharing will be achieved using SIP Session Handoff and Third-party Call Control respectively. Excerpt of HTTP messages can be sent in the form of SIP instant messages.

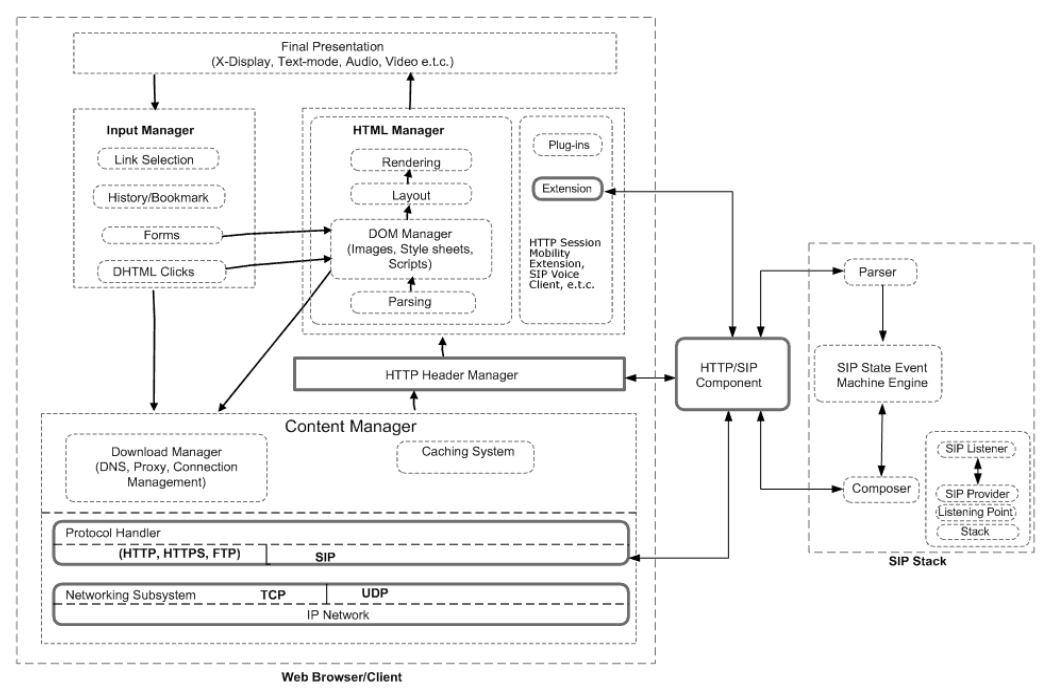

Fig. 1 The User Agent Client Architecture

\section{DISCUSSIONS}

\subsection{The User Agent Client Architecture}

Fig. 1 shows the modifications made to a User Agent Client Architecture. The bold rectangular boxes and the SIP stack represent parts of the architecture that will be extended and a new feature that will be integrated respectively. The Extension found in HTML Manager represents the User Interface (UI). When buttons on the UI are clicked, they trigger events such as registering the client and sending excerpt of the HTTP request/response header in form of SIP MESSAGE. These processes take place inside the HTTP/SIP Component block. SIP transactions are handled by the SIP Stack and works seamlessly with the HTTP/SIP Component which helps in building the SIP messages. The HTTP Header Manager is required to interact with the HTTP/SIP Component by providing the HTTP Request/Response excerpts. The Protocol Handler implements new SIP protocol alongside with the existing protocols such as HTTP and HTTPS. In the same manner, the Networking Subsystem integrates User Datagram Protocol (UDP) which is required during the SIP transactions. The other blocks such as Input Manager remain unmodified.

\subsection{Content Sharing and Session Transfer}

Content sharing refers to the process of sending same Universal Resource Locator (URL) to another User Agent. Session Transfer requires identifying which mechanism has been used to make HTTP stateful connection to a web server. It requires transferring session objects like cookies, URL and in some cases the entire HTTP Response unlike Content Sharing.

\subsection{The Open Problems}

There are many ways of making HTTP stateful. These include the use of session IDs or cookies, use of HTML hidden fields and URL encoding [10]. The UAC and the SIP AS will have the intelligence to detect what mechanism is used to make HTTP stateful. This feature will help in determining what excerpt of the HTTP header request needs to be transferred. For example, cookies or session ID and its associated URL can be encapsulated and sent between two UACs. In addition to detecting what mechanism is used, another open problem is how session transfer can be achieved when accessing a secured website that implements security measures like SSL.

\section{CONCLUSIONS}

This is a research work in-progress which aims at developing an extension with a SIP stack for a web browser and a SIP Application Server in order to achieve content sharing and session hand-off. While content sharing requires sending URL, most especially of non-secured websites, session transfer proves difficult considering the aforementioned open problems.

\subsection{References}

[1] D. Kristol el al, "HTTP State Management Mechanism," IETF RFC 2109, October 2000.

[2] B. Campbell et al, "Session Initiation Protocol (SIP) Extension for Instant Messaging," IETF RFC 3428, December 2002.

[3] R. Shacham et al, "Session Initiation Protocol (SIP) Session Mobility," Internet-Draft, July 2007.

[4] Sohel Q. Khan and Sprint-Nextel, "Experiences with Blending HTTP, RTSP and IMS," IEEE Communication Magazine, March 2007.

[5] Zap! The Mozilla SIP Client, http://www.croczilla.com/zap/.

[6] G. Canfora, G. Di Santo, G. Venturi, E. Zimeo and M.V. Zito, "Proxy-based Hand-off of Web Sessions for User Mobility," Proceedings of the Second Annual International Conference on Mobile and Ubiquitous Systems: Networking and Services (MobiQuitous '05), 2005.

[7] Ming-Deng Hsieh, Tsan-Pin Wang, Ching-Sung Tsai, Chien-Chao Tseng, "Stateful session handoff for mobile WWW," Information Sciences, Elsevier Science Press, volume 176, 2006, pp. 1241-1265.

[8] H. Song "Browser Session Preservation and Migration," In Poster Session of WWW 2002, Hawai, USA, 7-11 May, 2002, pp. 2.

[9] R. Fielding et al, "Hypertext Transfer Protocol - HTTP/1.1," IETF RFC 2616, June 1999.

[10] Hal Berghel, "Hijacking the Web," Communications of the ACM, Vol. 45 No. 4, April 2002. 